\title{
Plasma Procalcitonin in Patients with Ileus. Relations to Other Inflammatory Parameters
}

\author{
P. MARUNA ${ }^{1,2}$, R. FRAŠKOO $^{3}$, R. GÜRLICH ${ }^{3}$ \\ ${ }^{1}$ Institute of Pathological Physiology, ${ }^{2}$ Third Department of Internal Medicine, ${ }^{3}$ First Department \\ of Surgery, First Medical Faculty, Charles University, Prague, Czech Republic
}

Received March 20, 2007

Accepted April 30, 2007

On-line May 30, 2007

\begin{abstract}
Summary
Plasma procalcitonin (PCT) is a highly specific marker for the diagnosis of bacterial infections and sepsis. PCT levels are usually low in viral infections, chronic inflammation or postsurgical states. The purpose of this study was to characterize PCT plasma levels in patients with various types of ileus at preoperative stage, where the other inducing factors suchas a surgical stress are excluded. The prospective study was performed on 54 patients admitted to in-patient surgical department with a proven diagnosis of ileus. Patients were divided to three groups obstructive, vascular and paralytic ileus. Plasma levels of PCT (Kryptor analysis), TNF $\alpha$, IL-1 $\beta$, IL-6, cortisol (ELISA) and CRP (Kryptor ultrasensitive analysis) were estimated before any invasive procedure was realized. We demonstrated significant elevation of PCT in both obstructive ileus in adhesions and vascular ileus compared with healthy subjects $(p<0.01)$. PCT levels were not elevated in paralytic ileus. The regression coefficient was the highest for PCT and CRP $(r=0.78, p<0.01)$, for TNF $\alpha$ and IL-8 $(r=0.76, p<0.01)$ in vascular ileus. There was no significant correlation between PCT and other inflammatory parameters. The different types of ileus induce an elevation of plasma PCT levels and PCT shows itself as an acute phase reactant. The highest PCT concentrations were presented in patients with vascular ileus, whereas paralytic ileus revealed similar cytokine and PCT pattern as in healthy subjects. Plasma PCT estimation extended to a measurement of CRP and IL- 6 may become a useful complementary examination for diagnostics of acute abdomen in patients.
\end{abstract}

\section{Key words}

C-reactive protein $\bullet$ Ileus $\bullet$ Interleukin- $6 \bullet$ Procalcitonin $\bullet$ Tumor necrosis factor

\section{Corresponding author}

Pavel Maruna, Third Department of Internal Medicine of the First Medical Faculty UK, U nemocnice 1, 12000 Prague 2, Czech Republic. Fax: ++ 420 224919780. E-mail: maruna@LF1.cuni.cz

\section{Introduction}

When elevated levels of procalcitonin (PCT) were reported in patients with bacterial infection by Assicot et al. (1993), PCT became an important protein in the detection and differential diagnostics of inflammatory states. Now, PCT is a highly specific marker for the diagnosis of clinically relevant bacterial infections and sepsis (Castelli et al. 2004). One major advantage of PCT compared to both inflammatory cytokines and acute phase proteins (APP) is its early and specific increase in response to severe systemic bacterial infections (Maruna et al. 2000, Maruna 2003). Despite intensive research, a number of uncertainties still exist concerning the metabolism of this inflammatory PCT and its physiological role.

Regulations of thyroid and inflammatory PCT are fundamentally different. Bacterial infections induce a ubiquitous increase of the calcitonin gene 1 (CALC-1) expression on chromosome 11 and a constitutive release of PCT from all parenchymal tissues and differentiated cell types throughout the body (Morgenthaler et al. 2003). Quantitative analysis of PCT-mRNA expression shows a significant induction in all septic tissues. Hepatocytes and other parenchymal cells provide the principal source of circulating PCT in sepsis. The release of PCT is induced both directly via microbial antigens (e.g. endotoxin) and indirectly via an immune response, e.g. interleukin (IL)-1 $1 \beta$, tumor necrosis factor- $\alpha$ (TNF $\alpha$ ), and IL-6.

The both theoretical and clinical importance has a possibility of other non-bacterial (or generally noninfectious) factors inducing PCT synthesis in vivo. PCT

PHYSIOLOGICAL RESEARCH • ISSN 0862-8408 (print) • ISSN 1802-9973 (online)

(c) 2008 Institute of Physiology v.v.i., Academy of Sciences of the Czech Republic, Prague, Czech Republic

Fax +420 241062 164, e-mail: physres@biomed.cas.cz,www.biomed.cas.cz/physiolres 
levels are usually low in viral infections, chronic inflammatory disorders, abscesses or autoimmune processes. The non-infectious gastrointestinal conditions represent a point of interest due to their relations to portal venous system and liver as the supposed main source of inflammatory PCT (Russwurm et al. 2001).

The purpose of this study was to characterize PCT plasma levels in patients with various types of ileus at preoperative stage, where the other inducing factors as a surgical stress are excluded. Except of post-operative ileus, this is the first study aimed at plasma PCT changes induced by ileus. To get a more complex picture of immune response, we compared plasma PCT changes with four proinflammatory cytokines, which are related to PCT induction, cortisol, and C-reactive protein (CRP).

\section{Methods}

The prospective study was realized on the First Dept. of Surgery - Dept. of Abdominal Surgery of the First Faculty of Medicine in Prague in 2005-2007. The ethical committee of this institution approved this study protocol and informed consent was obtained from the subjects.

\section{Patients}

The study included 54 patients (24 males, 30 females, aged 25-71 years) admitted to the in-patient surgical department for clinical signs of ileus. The diagnosis of ileus was proved by following examinations according to standard guidelines for suspicion of acute abdomen. Patients with clinical signs of systemic or local inflammatory status, patients with a history of abdominal surgery in the last month, and patients with comorbidity or medications interacting with tested parameters (chronic inflammation, corticoids, antibiotics, and blood transfusion in recent history) were excluded from this study.

The patients were classified into groups according to a type of ileus - obstructive ileus in adhesions, vascular and paralytic ileus (Table 1). The submission was based on standard exam procedures (physical examination, ultrasonography, computed tomography scans, native X-ray exam) and/or a subsequent diagnostic laparotomy.

The control group for reference laboratory examinations consisted of 14 healthy persons ( 8 males, 6 females, 24-56 years).
PCT, cytokine, cortisol, and CRP analysis

Blood samples were drawn from cubital venous catheter before eventual invasive procedures. Blood samples of control group were drawn at baseline. For all measurements, $5 \mathrm{ml}$ of venous blood was taken into a vacutainer tube and immediately centrifuged at $5000 \mathrm{rpm}$ for $15 \mathrm{~min}$. Plasma was stored at $-80{ }^{\circ} \mathrm{C}$ until analysis. Plasma levels of IL-1 $\beta$, IL-6, IL-8, TNF $\alpha$, cortisol (ELISA, Immunities, France), CRP (Kryptor CRPultrasensitive analysis, BRAHMS, Germany) and PCT (Kryptor analysis, BRAHMS, Germany) were measured in duplicates. The intra- and inter-assay coefficients of variation were below $5 \%$.

\section{Statistical analysis}

The software package ANOVA ${ }^{\mathrm{TM}}$ for Windows, version 4.0, Insytec B.V, the Netherlands, was used for statistical evaluation. The Kolmogorov-Smirnov test was applied for the evaluation of the normality of the value distribution. As the data were normally distributed and independent, statistical analysis was performed using Student's t-test when comparing patient groups or controls vs. patients. The correlation among the monitored parameters was evaluated by the Pearson's correlation coefficient and the Spearman's rank correlation. Data were given as the mean \pm SD. Any $\mathrm{p}<0.05$ value was considered statistically significant.

Table 1. Patient groups and controls

\begin{tabular}{llll}
\hline Group & No. & M / F & age (years) \\
\hline Obstructive ileus & 28 & $11 / 17$ & $25-64$ \\
Vascular ileus & 16 & $7 / 9$ & $39-71$ \\
Paralytic ileus & 10 & $6 / 4$ & $28-61$ \\
Control group & 14 & $8 / 6$ & $24-56$ \\
\hline
\end{tabular}

\section{Results}

All patients with both obstructive and vascular ileus were submitted to surgical revision and all of them were operated at the day of admission. In addition, five patients with paralytic ileus were indicated to surgical revision for diagnostic uncertainty and a diagnosis was determined perioperatively. Mortality during the hospital stay was different among tested groups: obstructive ileus (3/28 patients), vascular ileus $(13 / 16)$, and paralytic ileus $(0 / 10)$. The average length of 


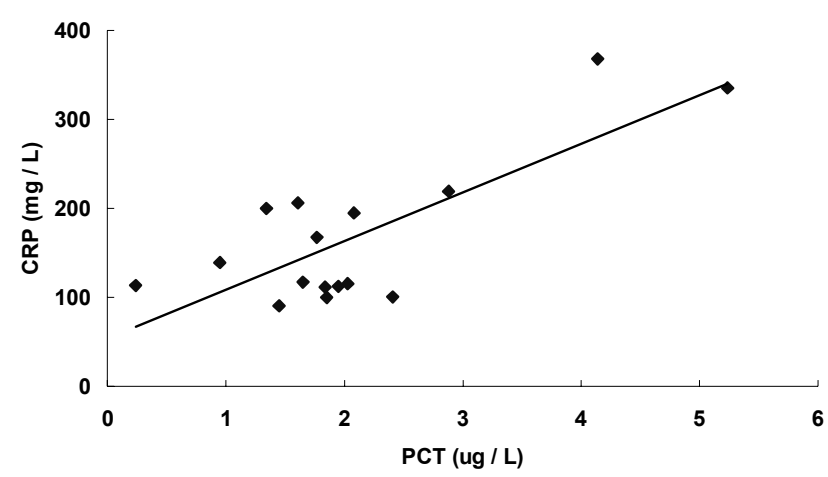

Fig. 1. Correlation between plasma concentrations of CRP and PCT in group 2 - vascular ileus $(n=16, r=0.78, p<0.01)$.

hospital stay was $13.2 \pm 5.4$ days in obstructive ileus, 16-27 days in three patients with vascular ileus and benign outcome; $8.1 \pm 2.4$ days in paralytic ileus. At the day of admission, white blood cell count was $8.2 \pm 2.6 \times$ $10^{3} / \mathrm{mm}^{3}$ in patients obstructive ileus, $10.1 \pm 2.1 \times$ $10^{3} / \mathrm{mm}^{3}$ in patients vascular ileus, and $7.2 \pm 1.9 \times$ $10^{3} / \mathrm{mm}^{3}$ in patients with paralytic ileus.

The levels of PCT, IL- $1 \beta$, IL-6, IL- 8, TNF $\alpha$, cortisol, and CRP are shown in Table 2. Student multiple comparison test confirmed significant differences $(p<0.05)$ between control group and patient groups. We demonstrated significant elevation of plasma PCT in both obstructive ileus in adhesions and vascular ileus compared with healthy subjects $(\mathrm{p}<0.05$ and $\mathrm{p}<0.01$, respectively). The highest concentrations of PCT, IL-1 $\beta$, IL-6, IL-8, cortisol, and CRP were found in patients with vascular ileus. In vascular ileus group, elevation of TNF $\alpha$, IL-6, IL-8, cortisol, and CRP was significant compared with control subjects $(p<0.05)$. In obstructive ileus group, the same parameters except of CRP were also significantly elevated compared with controls $(p<0.05) . T N F \alpha$ concentrations were highest in patients with obstructive ileus $(\mathrm{p}<0.05$ compared to control group). IL-1 $\beta$ was unable to distinguish patient groups from the control group. In contrast to other types of ileus, no tested inflammatory marker was significantly elevated in paralytic ileus. Among patient groups, significant differences were confirmed between group 1 (obstructive ileus) and group 2 (vascular ileus) for PCT $(\mathrm{p}<0.01)$ and CRP $(p<0.01)$. The same parameters differentiated group 2 (vascular ileus) and group 3 (paralytic ileus) $(\mathrm{p}<0.01)$. Other tested mediators did not distinguish different types of ileus.

In 3 patients with obstructive ileus and poor outcome, plasma levels of PCT ranged from 0.86 to $1.25 \mu \mathrm{g} / \mathrm{l}$, IL-6 levels were between 565-914 ng/l, and
CRP levels were between 18.0-52.6 mg/l. In 3 patients with vascular ileus and good outcome, we documented PCT concentrations between 0.24-1.65 $\mu \mathrm{g} / \mathrm{l}$, IL-6 212$388 \mathrm{ng} / \mathrm{l}$, and CRP $113.5-139.2 \mathrm{mg} / \mathrm{l}$.

The regression coefficients were highest for PCT and CRP ( $\mathrm{r}=0.78, \mathrm{p}<0.01), \mathrm{TNF} \alpha$ and IL-8 $(\mathrm{r}=0.76$, $\mathrm{p}<0.01)$, TNF $\alpha$ and IL- $8(\mathrm{r}=0.76, \mathrm{p}<0.01)$, and TNF $\alpha$ and $\mathrm{IL}-1 \beta(\mathrm{r}=0.67, \mathrm{p}<0.01)$ in group 2 (vascular ileus). Figure 1 shows the correlation between PCT and CRP in group 2. There was no significant correlation between PCT and any tested cytokine. Similarly, PCT did not correlate with cortisol in patient groups.

\section{Discussion}

We document in our study that various types of ileus are accompanied with an elevation of plasma PCT levels. The highest concentrations of PCT are present in patients with vascular ileus. The simultaneous elevation of TNF $\alpha$, IL- 6 and IL- 8 in this patient group indicates that mentioned cytokines play a role in PCT induction, and this mechanism is similar to other models of SIRSinduced PCT activation.

The generation of non-thyroid PCT is linked to both bacterial endotoxin and inflammatory cytokines. This was demonstrated on animal model by a rapid rise of PCT levels after the injection of endotoxin, or after the administration of TNF $\alpha$. Administration of IL-1, IL-2 and IL-6 also leads to an increase in PCT. Cytokines mediate PCT induction in various cell types, however, the mechanisms responsible for such an action are not known. The highest plasma levels of PCT are achieved in acute bacterial infections and sepsis. Little is known about other possible factors participating in PCT induction. Our previous study, in accordance with other authors, showed the transient PCT elevation after other non-infectious stimuli such as surgical trauma (Gürlich et al. 1999, Chachkhiani et al. 2005). The posttraumatic PCT increase is only moderate in most patients and peaked at day 1-2 after trauma.

As the liver is a possible main source of inflammatory PCT, the various types of ileus represent model situations of non-infectious gastrointestinal stimulus involving local cytokine production, portal venous system, and hepatocyte activation. Ileus, as a partial or complete non-mechanical blockage of the small and/or large intestine, may be the result of intestinal ischemia, abdominal wound infections, electrolyte imbalance (e.g. hypokalemia), anesthesia, surgery, 
Table 2. PCT, cytokines, cortisol, and CRP in patients with ileus and control group.

\begin{tabular}{llllllll}
\hline Group & $\begin{array}{l}\text { PCT } \\
(\boldsymbol{\mu g} / \mathbf{l})\end{array}$ & $\begin{array}{l}\mathbf{I L - 1} \boldsymbol{\beta} \\
\mathbf{( n g / \mathbf { l } )}\end{array}$ & $\begin{array}{l}\mathbf{I L - 6} \\
\mathbf{( n g} / \mathbf{l})\end{array}$ & $\begin{array}{l}\mathbf{I L - 8} \\
\mathbf{( n g / \mathbf { l } )}\end{array}$ & $\begin{array}{l}\mathbf{T N F a} \\
\mathbf{( n g} / \mathbf{l})\end{array}$ & $\begin{array}{l}\text { Cortisol } \\
(\mathbf{n g} / \mathbf{l})\end{array}$ & $\begin{array}{l}\text { CRP } \\
(\mathbf{m g} / \mathbf{l})\end{array}$ \\
\hline Obstructive & $0.75^{\mathrm{a}, \mathrm{b}, \mathrm{c}}$ & 4.4 & $547.0^{\mathrm{a}}$ & $46.6^{\mathrm{a}}$ & $55.9^{\mathrm{a}}$ & $786^{\mathrm{a}, \mathrm{c}}$ & $15.8^{\mathrm{b}}$ \\
ileus & \pm 0.26 & \pm 3.6 & \pm 261.3 & \pm 20.1 & \pm 31.5 & \pm 71 & \pm 12.05 \\
Vascular ileus & $2.09^{\mathrm{a}, \mathrm{d}}$ & 10.0 & $561.1^{\mathrm{a}}$ & $52.1^{\mathrm{a}}$ & $37.8^{\mathrm{a}}$ & $802^{\mathrm{a}, \mathrm{d}}$ & $168.3^{\mathrm{a}, \mathrm{d}}$ \\
& \pm 1.15 & \pm 8.5 & \pm 210.1 & \pm 16.5 & \pm 25.2 & \pm 106 & \pm 80.8 \\
Paralytic ileus & 0.17 & 5.9 & 310.7 & 30.8 & 16.0 & 612 & 7.4 \\
& \pm 0.07 & \pm 3.8 & \pm 136.8 & \pm 13.7 & \pm 14.2 & \pm 91 & \pm 6.24 \\
Control group & 0.21 & 4.3 & 222.8 & 18.5 & 10.1 & 491 & 4.7 \\
& \pm 0.11 & \pm 1.8 & \pm 73.3 & \pm 6.2 & \pm 6.4 & \pm 98 & \pm 2.4 \\
\hline
\end{tabular}

Data are mean values \pm SD. ${ }^{a}$ Statistical differences between patient group and control group $(p<0.05),{ }^{b}$ Statistical differences between obstructive ileus and vascular ileus $(p<0.05),{ }^{c}$ Statistical differences between obstructive ileus and paralytic ileus $(p<0.05),{ }^{d}$ Statistical differences between vascular ileus and paralytic ileus $(p<0.05)$.

dysbalance of intestinal nerve supply, or metabolic diseases. There are different types of intestinal obstructions, mechanical and non-mechanical including vascular and paralytic ileus.

Several studies tested PCT changes in postoperative ileus stage. After abdominal surgery, a physiological temporary absence of the normal intestinal contractile movements occurs (Maruna et al. 2005, Fraško et al. 2004). This type of ileus spontaneously resolves within 2-3 days after bowel motility returns to normal. This is a markedly different situation from our patients, because observed PCT induction during postoperative ileus is a part of a systemic inflammatory response syndrome (SIRS) to surgical stress. Several studies including our previous observations (Maruna et al. 2002) described the kinetics and the amount of PCT induced after elective surgery and trauma. The up-regulation and release of proinflammatory cytokines after surgery has been shown to play an important role in postoperative ileus. For example, colorectal surgery is associated with higher levels of IL-6 than any other operation and is often associated with postoperative ileus. The postoperative ileus was described on animal models (Wehner et al. 2005). Mild intestinal manipulation in rodents initiates the up-regulation of proinflammatory cytokines, and increases the release of kinetically active mediators (nitric oxide and prostaglandins), which results in the recruitment of leukocytes and suppression of motility.

Our clinical study is the first observation evaluating plasma PCT changes induced by ileus in nonpost-surgical period. The diagnosis of ileus was based on medical history, typical symptoms, physical exam, and testing including X-ray, ultrasonography and/or computed tomography scan, eventually colonoscopy. Blood sample collection was realized before any invasive procedure to check off other potential factors interacting with stress humoral response. Similarly, other SIRS modifying factors as blood transfusion were excluded. Ileus may be associated with a peritoneal infection influencing both local and systemic cytokine responses, therefore these patients had to be eliminated from our stuided groups. Patients with clinical or laboratory signs of abdominal or other inflammation were also excluded from this study.

Both obstructive and vascular ileus is accompanied by an elevation of PCT. However, its concentrations do not reach levels typical for systemic inflammation. What is a mechanism of PCT induction in patients with ileus? Soybel and Zinner (2003), Wehner et al. (2005) and other revealed that ileus is associated with the locally increased expression of inflammatory cytokines and chemokines, a leukocyte infiltration into the muscularis, and the release of mediators from resident and infiltrating leukocytes. These mediators may directly inhibit intestinal smooth muscle contractility and accomplish the course of ileus. Our study documents both relatively mild elevations of plasma proinflammatory cytokines and insignificant correlation between cytokine and PCT plasma levels. These findings suggest that cytokine responses take place in a local milieu without a more remarkable systemic cytokine network activation. On the other hand, this intestinal production of inflammatory mediators may stimulate PCT formation in both intestinal macrophages and hepatocytes via portal system.

Our results displayed vascular ileus as the most 
intensive stimulus for both systemic cytokine network and PCT production among various types of ileus. Compromised mesenteric blood flow leading to progressive mesenteric ischemia is associated with ischemic changes of the bowel wall. Atherosclerosis of the aorta and/or unpaired visceral branches is the main cause of an acute disturbance of mesenteric circulation. Baeshko et al. (2005) showed that in $87 \%$ cases the causes of acute mesenteric ischemia were occlusive disease of intestinal vessels due to thrombosis and embolism of unpaired visceral branches of the abdominal aorta. We assume that PCT elevation is an indirect effect of mesenteric ischemia mediated via cytokine activation. Intestinal ischemia causes local injury that is multifactorial and essentially inflammatory in nature. Cavriani et al. (2007) recently published experimental findings on intestinal ischemia/reperfusion model in rats. The ischemia/reperfusion caused a significant increase of the serum levels of IL-1 $\beta$ and IL-10 in rats. Several studies revealed a similar role of IL-6, recently by Yamaguchi and Uchida (2007). The observation that the thoracic duct ligation blunted the serum release of IL-1 $\beta$ and IL-10 documents the participation of both portal venous system and thoracic lymph duct on this systemic cytokine overflow (Cavriani et al. 2007). The proinflammatory cytokines represent links between tissue ischemia and PCT elevation. Administration of TNF $\alpha$, IL-1 $\beta$, and IL-6 leads to an increase in PCT in experimental animals. In spite of the large body of data, it is still uncertain how and from which tissues PCT is released into the circulation. PCTmRNA expression was found in liver, colon, skin, spleen, pancreas and many other organs (Morgenthaler et al. 2003). The lack of appropriate experimental findings makes some conclusions difficult. Both the main cell source of PCT and detailed pathogenesis of PCT induction in different types of ileus are unknown, yet, and their understanding needs subsequent studies.

Maisner et al. (2006) showed in multiple-trauma patients that the higher concentrations of PCT correlate with a higher frequency of various complications, including sepsis and infection, and the PCT specificity exceeded that of CRP. Our patients with obstructive, vascular and paralytic ileus differed markedly in their outcome. Particularly vascular ileus was characterized by very high mortality. In vascular ileus group, the only three patients with good prognosis showed the lowest plasma PCT levels among patients of this group. Nevertheless, low number of survivals did not allow any definite conclusions.

We documented the absence of CRP elevation in patients with obstructive ileus, whereas IL-6 was elevated in this group and its levels were similar to concentrations found in vascular ileus. This IL-6/CRP dissociation may be hypothesized only and its clarification needs repeated evaluation of mentioned proteins during a longer period. IL-6 is a main inductor of CRP as a typical 2nd type APP, but the dynamics of plasma CRP and IL-6 are different in relation to their successive induction, dissimilar plasma half-life and a role of other CRP-inducing factors. The possible explanation is a modulating role of TNF $\alpha$ and IL$1 \beta$ in CRP induction. These two cytokines are not necessary for CRP activation in vitro. However, they may amplify IL-6 mediated 2nd type APP activation in vivo. Interactions among cytokines, PCT and APP take place on both local and systemic levels. Plasma 'overflow' of IL-1 $\beta$ and other cytokines during SIRS does not reflect their tissue expression directly. This explanation may be assumed for only mild IL-1 $\beta$ elevation bellow statistical significance in all tested patient groups. Prevailing local activities of CRP-inducing cytokines may also partially explain disproportion of both CRP and cytokine plasma concentrations.

We conclude that the different types of ileus induce an elevation of plasma PCT levels. The highest PCT concentrations were presented in patients with vascular ileus whereas paralytic ileus revealed similar inflammatory pattern like in healthy subjects. Plasma PCT estimation extended to a measurement of CRP and IL-6 may become a useful complementary examination for diagnostics of acute abdomen in patients.

\section{Conflict of Interest}

There is no conflict of interest.

\section{Acknowledgements}

This work was supported by grants MSM0021620819 and IGA Ministry of Health NR/8152-3

\section{References}

ASSICOT M, GENDREL D, CARSIN H, RAYMOND J, GUILBAUD J, BOHUON C: High serum procalcitonin concentrations in patients with sepsis and infection. Lancet 341: 515-518, 1993.

BAESHKO AA, KLIMUK SA, IUSHKEVICH VA: Causes and features of involvement of the intestine and its vessels in acute disturbance of mesenterial circulation. Khirurgiia (Mosk) 4: 57-63, 2005. 
CHACHKHIANI I, GURLICH R, MARUNA P, FRASKO R, LINDNER J: The postoperative stress response and its reflection in cytokine network and leptin plasma levels. Physiol Res 54: 279-285, 2005.

CASTELli GP, POGNANI C, MEISNER M, STUANI A, BELLOMI D, SGARBI L: Procalcitonin and C-reactive protein during systemic inflammatory response syndrome, sepsis and organ dysfunction. Crit Care 8: R234R242, 2004.

CAVRIANI G, DOMINGOS HV, OLIVEIRA-FILHO RM, SUDO-HAYASHI LS, VARGAFTIG BB, DE LIMA WT: Lymphatic thoracic duct ligation modulates the serum levels of IL-1 $\beta$ and IL-10 after intestinal ischemia/reperfusion in rats with the involvement of tumor necrosis factor alpha and nitric oxide. Shock 27: 209-213, 2007.

GÜRLICH R, MARUNA P, ČERMÁK J: Use of procalcitonin in surgery. Rozhl Chir 78: 292-294, 1999.

GÜRLICH R, MARUNA P, FRAŠKO R: Therapeutic approach for treatment of postoperative ileus. Rozhl Chir 83: 562-566, 2004.

MARUNA P, NEDĚLNÍKOVÁ K, GÜRLICH R: Physiology and genetics of procalcitonin. Physiol Res 49: S57-S61, 2000.

MARUNA P, GÜRLICH R, FRAŠKO R, CHACHKHIANI I, MARUNOVÁ M, OWEN K, PEŠKOVÁ M: Procalcitonin in the diagnosis of postoperative complications. Sb Lek 103: 283-295, 2002.

MARUNA P: Prokalcitonin. Triton, Prague, 2003.

MARUNA P, GÜRLICH R, FRAŠKO R: Pathophysiology of postoperative dysfunctions of the intestinal motility. Rozhl Chir 84: 356-362, 2005.

MEISNER M, ADINA H, SCHMIDT J: Correlation of procalcitonin and C-reactive protein to inflammation, complications, and outcome during the intensive care unit course of multiple-trauma patients. Crit Care 10: R1, 2006.

MORGENTHALER NG, STRUCK J, CHANCERELLE Y, WEGLOHNER W, AGAY D, BOHUON C, SUAREZDOMENECH V, BERGMANN A, MULLER B: Production of procalcitonin in non-thyroidal tissue after LPS injection. Horm Metab Res 35: 290-295, 2003.

RUSSWURM S, STONANS I, STONANE E, WIEDERHOLD M, LUBER A, ZIPFEL PF, DEIGNER HP, REINHART K: Procalcitonin and CGRP-1 mRNA expression in various human tissues. Shock 16: 109-112, 2001.

SOYBEL DI, ZINNER MJ: Ileus and the macrophage. Ann Surg 237: 316-318, 2003.

WEHNER S, SCHWARZ NT, HUNDSDOERFER R, HIERHOLZER C, TWEARDY DJ, BILLIAR TR, BAUER AJ, KALFF JC: Induction of IL-6 within the rodent intestinal muscularis after intestinal surgical stress. Surgery 137: 436-446, 2005.

YAMAGUCHI M, UCHIDA M: Alpha-lactalbumin suppresses interleukin-6 release after intestinal ischemia/reperfusion via nitric oxide in rats. Inflammopharmacology 15: 43-47, 2007. 